\title{
Stopband-Extended Balanced Bandpass Filter Using Coupled Stepped-Impedance Resonators
}

\author{
Chung-Hwa Wu, Student Member, IEEE, Chi-Hsueh Wang, Member, IEEE, and Chun Hsiung Chen, Fellow, IEEE
}

\begin{abstract}
A novel fourth-order balanced bandpass filter is proposed based on the half-wavelength $(\lambda / 2)$ stepped-impedance resonators (SIRs). By properly adjusting the parameters of each SIR, the proposed filter may be made compact and its stopband may also be extended simultaneously. Specifically, a balanced filter with acceptable common-mode rejection is implemented with its differential-mode and common-mode stopbands extended up to $5.5 f_{0}^{d}$, where $f_{0}^{d}$ is the center frequency of differential-mode passband.
\end{abstract}

Index Terms-Balanced filter (BPF), coupled-resonator BPF, stepped-impedance resonator (SIR), stopband extension.

\section{INTRODUCTION}

B ALANCED circuits play an important role in building a modern communication system particularly under the trend of system-on-chip (SOC). Balanced circuits with differential operation show higher immunity to environmental noise when compared with unbalanced circuits with single-ended signaling. To complete a balanced system, one needs to develop the balanced filters accordingly. A well-designed balanced bandpass filter (BPF) should exhibit the desired differential-mode frequency response and should also be capable of reducing the common-mode signal at the same time. However, pervious works on balanced filters are rather limited [1]-[3].

Recently, a fourth-order balanced coupled-line BPF using parallel-coupled-line structures was proposed in [3], which presents both high selectivity and good common-mode rejection within the differential-mode passband. However, this filter still has several drawbacks, such as requiring via-hole process and exhibiting a spurious common-mode passband around $1.75 f_{0}^{d}$. In this study, a novel fourth-order balanced BPF based on $\lambda / 2$ stepped-impedance resonators (SIRs) is proposed to realize the desired differential-mode characteristics in addition to overcoming the shortcomings in [3]. First, by making good use of structure symmetry to present a perfect electric conductor (PEC) wall along the symmetric-line, the undesired via-hole process may be avoided. Second, by properly adjusting the impedance and length ratios of each SIR, the proposed filter may be made compact and its spurious passbands for both differential-mode and common-mode excitations may be pushed up to a higher frequency so as to extend the bandwidth of stopband [4].

Manuscript received December 18, 2006; revised February 27, 2007. This work was supported by the National Science Council of Taiwan, R.O.C., under Grants NSC 95-2752-E-002-001-PAE, NSC 95-2219-E-002-008, and NSC 95-2221-E-002-196.

The authors are with the Department of Electrical Engineering and Graduate Institute of Communication Engineering, National Taiwan University, Taipei 106, Taiwan, R.O.C. (e-mail: chchen@ew.ee.ntu.edu.tw).

Digital Object Identifier 10.1109/LMWC.2007.899311

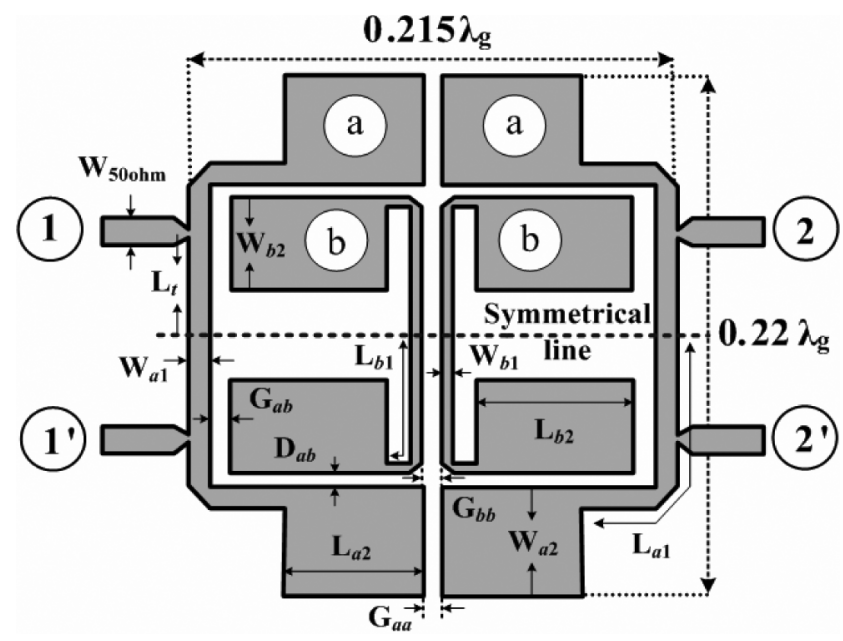

Fig. 1. Physical layout of the proposed fourth-order balanced filter using symmetric $\lambda / 2 \operatorname{SIRs}\left(W_{50 \Omega}=1.9 \mathrm{~mm}, W_{a 1}=1.5 \mathrm{~mm}, W_{a 2}=7.6 \mathrm{~mm}, W_{b 1}=\right.$ $0.8 \mathrm{~mm}, W_{b 2}=6.8 \mathrm{~mm}, L_{a 1}=17.1 \mathrm{~mm}, L_{a 2}=9.5 \mathrm{~mm}, L_{b 1}=11.9 \mathrm{~mm}$ $L_{b 2}=11.3 \mathrm{~mm}, L_{t}=7.2 \mathrm{~mm}, G_{a a}=1.2 \mathrm{~mm}, G_{b b}=1.4 \mathrm{~mm}, G_{a b}=$ $0.8 \mathrm{~mm}, D_{a b}=0.4 \mathrm{~mm}$ )

\section{BALANCEd FiLter Structure}

The proposed fourth-order balanced filter illustrated in Fig. 1 is composed of four symmetric $\lambda / 2$ SIRs which are similar to the ones adopted in [5]. Different from [5] which used the unbalanced feed structure with single-ended signaling, the proposed balanced filter has used the balanced feed structure for giving extremely different boundary conditions with differential operation. Specifically, under differential-mode and common-mode excitations, one may present the perfect electric wall (PEC) and the perfect magnetic wall (PMC), respectively, along the symmetric-line of the balanced filter structure. Thus, it is possible to reduce the level of common-mode noise in addition to possessing the desired bandpass frequency response in differential-mode operation.

Each $\lambda / 2$ SIR in Fig. 1, as illustrated in Fig. 2(a), is symmetric and has different characteristic impedances $Z_{i 1}$ and $Z_{i 2}$ and lengths $L_{i 1}$ and $L_{i 2}$, where the subscript $i$ denotes the resonators $\mathrm{a}$ and $\mathrm{b}$, respectively. The associated parameters such as the impedance ratio $R_{i}$ and the length ratio $\alpha_{i}$ are defined by

$$
R_{i}=\frac{Z_{i 2}}{Z_{i 1}}, \quad \alpha_{i}=\frac{L_{i 2}}{\left(L_{i 1}+L_{i 2}\right)} \cdot i=a, b .
$$

Under differential-mode operation, a virtual-short (PEC) would appear along the symmetric-line, therefore each resonator, resonating at $f_{0}^{d}$, may be treated as a shorted $\lambda / 4 \operatorname{SIR}$ as shown in Fig. 2(b). Alternatively, under common-mode 


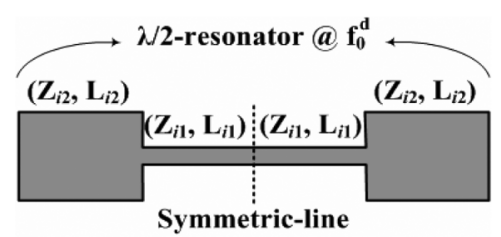

(a)

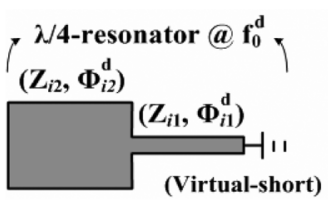

(b)

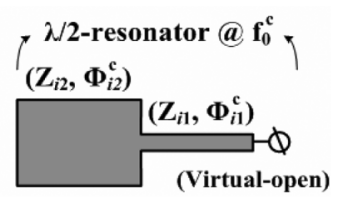

(c)
Fig. 2. (a)Basic SIR structure, (b) differential-mode equivalent-half-circuit, and (c) common-mode equivalent-half-circuit.

operation, a virtual-open (PMC) would present along the symmetric-line, thus each resonator, resonating at $f_{0}^{c}\left(f_{0}^{c}>f_{0}^{d}\right)$, may be treated as a $\lambda / 2$ resonator with both ends opened as shown in Fig. 2(c).

The balanced BPF shown in Fig. 1 is fabricated in the microstrip structure on the FR4 substrate $\left(\varepsilon_{r}=4.4, h=1 \mathrm{~mm}\right.$, $\tan \delta=0.02)$. It is designed with a differential-mode center frequency $f_{0}^{d}$ at $1 \mathrm{GHz}$ and $3-\mathrm{dB}$ fractional bandwidth (FBW) of $10 \%$. For characterization, the balanced structure, as a four-port device, is first measured by the Agilent E5071B network analyzer to give the standard four-port S-parameters $S^{\text {std }}$. Then the two-port differential-mode and common-mode $S$-parameters, $S^{\text {dd }}$ and $S^{\text {cc }}$, may be extracted from the four-port $S$-parameters $S^{\text {std }}$ as given in [3] and [6].

\section{Design of The Stopband-Extended BALANCED FILTER}

To design a stopband-extended balanced filter with desired differential-mode frequency response, the physical dimensions of each resonator must be determined first. According to [7], the differential-mode stopband response is mainly determined by the intercoupled resonators (resonators $b$ ). To push the first differential-mode spurious harmonic to a higher frequency such as $5.5 f_{0}^{d}$, the parameters $R_{b}=0.25$ and $\alpha_{b}=0.5$ should be chosen for the resonators $b$. For the SIR to possess the fundamental resonance frequency at $f_{0}^{d}(=1 \mathrm{GHz})$, the physical dimensions $\left(W_{b 1}\right.$, $W_{b 2}, L_{b 1}$, and $L_{b 2}$ ) of resonators $b$ should properly be selected. The dimensions ( $W_{a 1}, W_{a 2}, L_{a 1}$, and $L_{a 2}$ ) of input/output (I/O) resonators (resonators $a$ ) are then arranged so that the filter may be made compact and an additional cross-coupled path between I/O resonators may be established.

Then, according to the specification of the quasi-elliptic response with $f_{0}^{d}=1 \mathrm{GHz}$ and $3 \mathrm{db}-\mathrm{FBW}=10 \%$, the differential-mode coupling coefficients and I/O external quality factors calculated from [8] may be expressed as

$$
\begin{aligned}
Q_{e}^{\mathrm{dd}} & =\frac{g_{0} g_{1}}{F B W}=9.55, \quad M_{a b}^{\mathrm{dd}}=\frac{F B W}{\sqrt{g_{1} g_{2}}}=0.087 \\
M_{b b}^{\mathrm{dd}} & =\frac{F B W \cdot J_{2}}{g_{2}}=0.0767 \\
M_{a a}^{\mathrm{dd}} & =\frac{F B W \cdot J_{1}}{g_{1}}=-0.017
\end{aligned}
$$

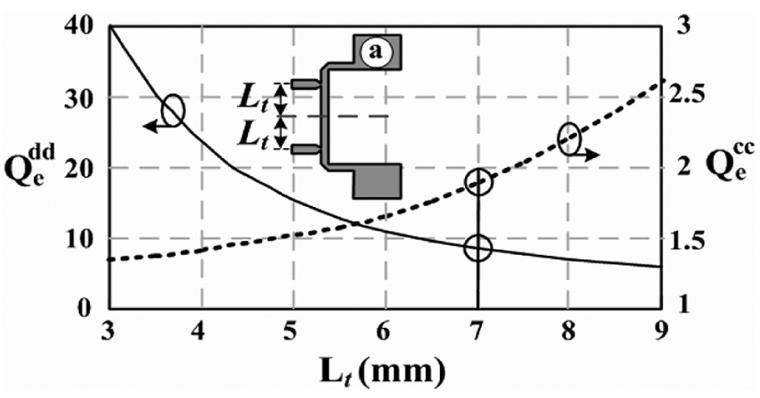

Fig. 3. Simulated differential-mode and common-mode external quality factors against the tap position of $\mathrm{I} / \mathrm{O}$ resonators.

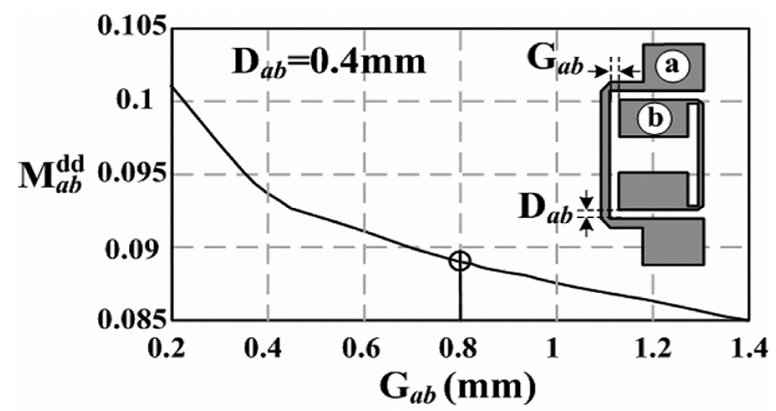

(a)

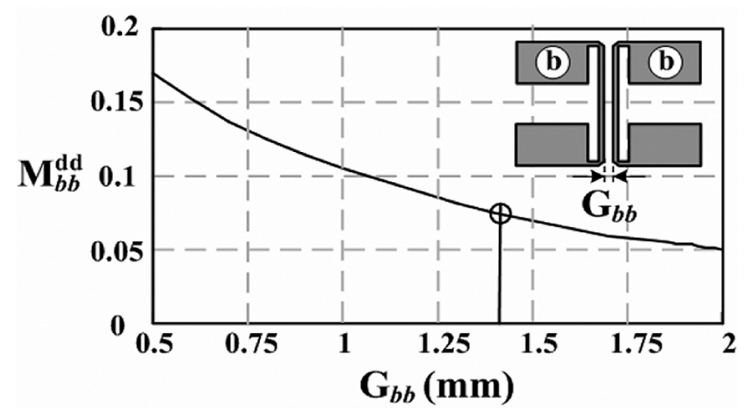

(b)

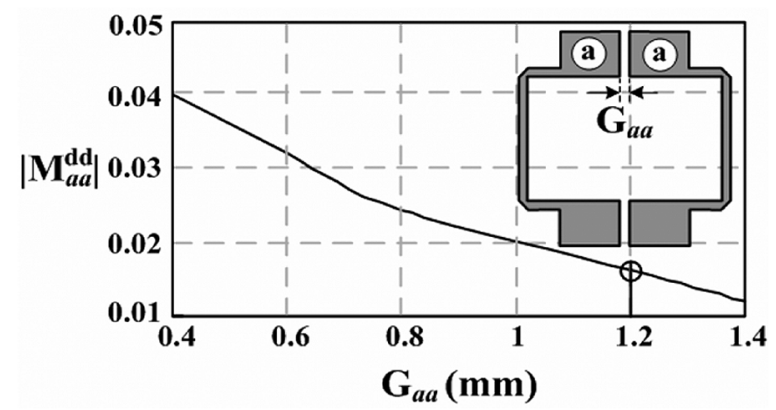

(c)

Fig. 4. Simulated differential-mode coupling coefficients versus the gaps between adjacent resonators: (a) mixed coupling, (b) magnetic coupling, and (c) electric coupling.

where $g_{1}, g_{2}, J_{1}$, and $J_{2}$ are the element values of the lowpass prototype filter. Instead of using the four-port to two-port conversion technique [6] used in measurement, the required differential-mode and common-mode parameters $M_{i j}$ and $Q_{e}$ are extracted from the corresponding differential-mode and common-mode excitation circuits, which have the termination impedances $\left(2 Z_{o}\right)$ and $\left(Z_{o} / 2\right)$, respectively. In this study, the 


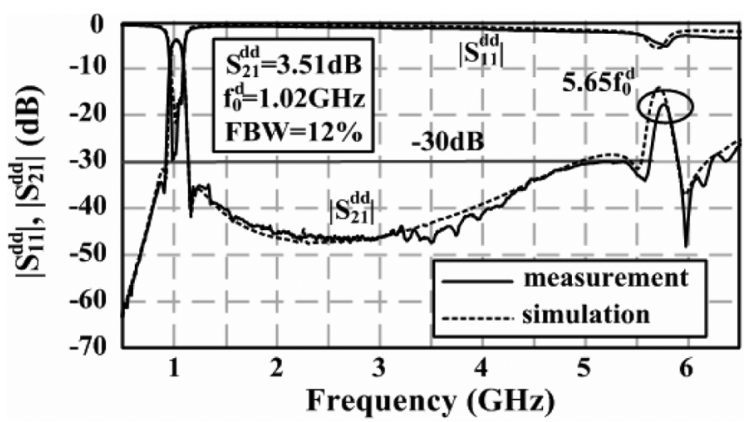

(a)

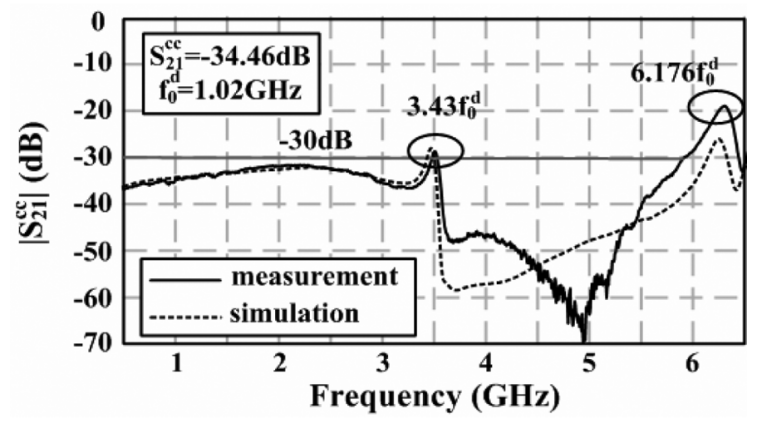

(b)

Fig. 5. Wideband measured and simulated frequency responses of the proposed fourth-order balanced filter shown in Fig. 1: (a) differential-mode response and (b) common-mode response.

extraction process is accomplished by the fullwave simulator ADS Momentum. Once the physical dimensions of each resonator are determined, the dimensions associated with the tap position $L_{t}$ of I/O resonators (resonators $a$ ) and the gaps $\left(G_{a a}, G_{b b}, G_{a b}\right.$, and $\left.D_{a b}\right)$ between adjacent resonators may be obtained by using the design curves as shown in Figs. 3 and 4.

\section{FILTER RESPONSES}

\section{A. Differential-Mode Response}

The wideband measured and simulated differential-mode responses of the proposed balanced filter (Fig. 1) are illustrated in Fig. 5(a). Good agreement between measured and simulated results is obtained. For the differential-mode response, the center frequency is at $1.02 \mathrm{GHz}$, with a minimum insertion-loss of $3.51 \mathrm{~dB}$ and a bandwidth of $12 \%$. Moreover, with the proper arrangement of impedance ratio $R_{b}$ and length ratio $\alpha_{b}$ of resonators $b$, the differential-mode spurious passband has been pushed up to $5.65 f_{0}^{d}$. The implemented filter is compact and has a size of $33.4 \mathrm{~mm} \times 36.2 \mathrm{~mm}\left(0.215 \lambda_{g} \times 0.22 \lambda_{g}\right)$, where $\lambda_{g}$ is the guided wavelength of the microstrip at the passband center frequency.

\section{B. Common-Mode Response}

Under common-mode excitation, the signal along the main path (from input resonator $a$ through inter-coupled resonators $b$ and then to output resonator $a$ ) is relatively weak like the one illustrated in [3]. Alternatively, the signal along the cross-coupled path (from input resonator $a$ directly to output resonator $a$ ) is relatively strong, therefore, the common-mode response is mainly determined by the coupling directly between I/O resonators. Moreover, the corresponding common-mode external quality factor $\left(Q_{e}^{c c}\right)$ is quite small, which presents an unmatched termination around $f_{0}^{d}$. Therefore, only an acceptable common-mode suppression may be obtained around $f_{0}^{d}$

The wideband measured and simulated common-mode responses are shown in Fig. 5(b). The common-mode signal is suppressed with a level of $-34.46 \mathrm{~dB}$ around $f_{0}^{d}$, and almost below $-30 \mathrm{~dB}$ from 0.5 to $6 \mathrm{GHz}$. The common-mode signal has a suppressed spurious response around $3.43 f_{0}^{d}$ which is associated with the first spurious harmonic of the resonator $b$. This spurious response has been suppressed due to the relatively weak signal along the main path and the poor $Q_{e}^{\mathrm{cc}}$ value associated with I/O resonators. Note that the open stub associated with tap position $L_{t}$ has created an additional transmission zero at $5 \mathrm{GHz}$ so that the common-mode rejection is improved around this stopband.

\section{CONCLUSION}

In this letter, a novel fourth-order balanced BPF using symmetric $\lambda / 2$ SIRs is proposed. Design procedures and the corresponding frequency responses are also carefully examined. With the adoption of symmetric coupled-resonator structures, it is possible to obtain the desired differential-mode frequency response and also to avoid the undesired via-hole process. Meanwhile, by the proper arrangement of impedance and length ratios of each resonator, a compact balanced filter is implemented with its differential-mode and common-mode stopbands extended up to $5.5 f_{0}^{d}$.

\section{REFERENCES}

[1] A. Ziroff, M. Nalezinski, and W. Menzel, "A 40 GHz LTCC receiver module using a novel submerged balancing filter structure," in Proc. Rad. Wireless Conf., 2003, pp. 151-154.

[2] Y.-S. Lin and C. H. Chen, "Novel balanced microstrip coupled-line bandpass filters," in In Proc. URSI Int. Electromag. Theory Symp., 2004, pp. 567-569.

[3] C.-H. Wu, C.-H. Wang, and C. H. Chen, "Novel balanced coupled-line bandpass filters with common-mode noise suppression," IEEE Trans. Microw. Theory Tech., vol. 55, no. 2, pp. 287-295, Feb. 2007.

[4] M. Sagawa, M. Makimoto, and S. Yamashita, "Geometrical structures and fundamental characteristics of microwave stepped-impedance resonators," IEEE Trans. Microw. Theory Tech., vol. 45, no. 7, pp. 1078-1085, Jul. 1997.

[5] S.-Y. Lee and C.-M. Tsai, "New cross-coupled filter design using improved hairpin resonators," IEEE Trans. Microw. Theory Tech., vol. 48, no. 12 , pp. 2482-2490, Dec. 2000.

[6] D. E. Bockelman and W. R. Eisenstant, "Combined differential and common-mode scattering parameters: Theory and simulation," IEEE Trans. Microw. Theory Tech., vol. 43, no. 7, pp. 1530-1539, Jul. 1995.

[7] S.-C. Lin, P.-H. Deng, Y.-S. Lin, C.-H. Wang, and C. H. Chen, "Wide-stopband microstrip bandpass filters using dissimilar quarter-wavelength stepped-impedance resonators," IEEE Trans. Microw. Theory Tech., vol. 54, no. 3, pp. 1011-1018, Mar. 2006.

[8] J. S. Hong and M. J. Lancaster, Microstrip Filters for RF/Microwave Applications. New York: Wiley, 2001. 